\title{
Fault-tolerant analysis of a class of networks
}

\author{
Jun-Ming $\mathrm{Xu}^{\mathrm{a}, *}$, Qiang Zhu ${ }^{\mathrm{b}}$, Min $\mathrm{Xu}^{\mathrm{c}, 1}$ \\ a Department of Mathematics, University of Science and Technology of China, Hefei, Anhui 230026, China \\ b Department of Mathematics, XiDian University, Xi'an, Shanxi 710071, China \\ c School of Mathematical Sciences, Beijing Normal University, Beijing 100875, PR China
}

Received 28 February 2007; received in revised form 9 April 2007; accepted 10 April 2007

Available online 25 April 2007

Communicated by L. Boasson

\begin{abstract}
In this paper, we explore the 2-extraconnectivity of a special class of graphs $G\left(G_{0}, G_{1} ; M\right)$ proposed by Chen et al. [Y.-C. Chen, J.J.M. Tan, L.-H. Hsu, S.-S. Kao, Super-connectivity and super edge-connectivity for some interconnection networks, Applied Mathematics and Computation 140 (2003) 245-254]. As applications of the results, we obtain that the 2-extraconnectivities of several well-known interconnection networks, such as hypercubes, twisted cubes, crossed cubes, Möbius cubes and locally twisted cubes, are all equal to $3 n-5$ when their dimension $n$ is not less than 8 . That is, when $n \geqslant 8$, at least $3 n-5$ vertices must be removed to disconnect any one of these $n$-dimensional networks provided that the removal of these vertices does not isolate a vertex or an edge.
\end{abstract}

(c) 2007 Elsevier B.V. All rights reserved.

Keywords: Combinatorial problems; Connectivity; Extraconnectivity; Hypercube

\section{Introduction}

It is well known that when the underlying topology of an interconnection network is modeled by a connected graph $G=(V, E)$, where $V$ is the set of processors and $E$ is the set of communication links in the network, the connectivity $\kappa(G)$ and the edge-connectivity $\lambda(G)$ are the two important features determining reliability and fault tolerance of the network [18]. These two parameters, however, have a obvious deficiency, that is,

\footnotetext{
The work was supported by NNSF of China (Nos. 10671191 and 10626053).

* Corresponding author.

E-mail address: xujm@ustc.edu.cn (J.-M. Xu).

1 Part of the work was done while the author was working at Inst. of Applied Math., Chinese Academy of Sciences, Beijing, China.
}

they tacitly assume that all vertices adjacent to or all edges incident with the same vertex of $G$ can potentially fail at the same time, which happens almost impossible in practical applications of networks. In other words, in the definitions of $\kappa$ and $\lambda$, absolutely no restrictions are imposed on the components of $G-S$. Consequently, these two measurements are inaccurate for large-scale processing systems in which all processors adjacent to or all links incident with the same processor cannot fail at the same time. To compensate for this shortcoming, it would seem natural to generalize the notion of the classical connectivity by imposing some conditions or restrictions on the components of $G-S$. Haray [10] first considered this problem by introducing the concept of the conditional connectivity.

Given a graph $G$ and a graph-theoretical property $\mathscr{P}$, he defined the conditional connectivity $\kappa(G ; \mathscr{P})$ (resp., 
edge-connectivity $\lambda(G ; \mathscr{P}))$ as the minimum cardinality of a set of vertices (resp., edges), if any, whose deletion disconnects $G$ and every remaining component has property $\mathscr{P}$. Clearly, $\kappa(G)$ (resp., $\lambda(G)$ ) is a special case of $\kappa(G ; \mathscr{P})$ (resp., $\lambda(G ; \mathscr{P}))$ when no condition is restricted to $\mathscr{P}$. The existence and value of $\kappa(G ; \mathscr{P})$ $(\lambda(G ; \mathscr{P}))$ varies depending on the different choice of the property $\mathscr{P}$.

Fàbrega and Fiol [9] considered $\kappa\left(G ; \mathscr{P}_{h}\right)$ (resp., $\left.\lambda\left(G ; \mathscr{P}_{h}\right)\right)$ for a non-negative integer $h$ and a graph $G$, where $\mathscr{P}_{h}$ is the property of having more than $h$ vertices. They called this type of connectivity as the $h$-extraconnectivity (resp., $h$-edge-extraconnectivity) of $G$, denoted by $\kappa_{h}(G)$ (resp., $\lambda_{h}(G)$ ). In other words, $\kappa_{h}(G)$ (resp., $\lambda_{h}(G)$ ) is the minimum cardinality of a set of vertices (resp., edges) of $G$, if any, whose deletion disconnects $G$ and every remaining component has more then $h$ vertices.

Clearly, $\kappa_{0}(G)=\kappa(G)$ and $\lambda_{0}(G)=\lambda(G)$ for any graph $G$ if $G$ is not a complete graph. Thus, the $h$-extraconnectivity is a generalization of the classical connectivity and can provides more accurate measures for the reliability and the tolerance of a large-scale parallel processing system, and so has received much research attention (see, for example, [6-8,12-16,20-22,24,2628]) for $h=1$ in recent years. However, a few of results for $h \geqslant 2$ are known in the present literature, for example, [29].

Fàbrega et al. [9] studied the extraconnectivity of some class of graphs with large girth. But the girth of most of the well-known interconnection networks is small. Thus we are interested in the study of the extraconnectivity of the well-known interconnection networks with small girth. In this paper, we consider a class of graphs, denoted by $G\left(G_{0}, G_{1} ; M\right)$ and proposed by Chen et al. [3]. In [23], $\mathrm{Xu}$ and Wang studied the 1extraconnectivity and the 1-edge-extraconnectivity of $G\left(G_{0}, G_{1} ; M\right)$. In [29], Zhu et al. studied the 2-edge-extraconnectivity of $G\left(G_{0}, G_{1} ; M\right)$. In this paper, we study the 2-extraconnectivity of $G\left(G_{0}, G_{1} ; M\right)$. As applications, we obtain the 2-extraconnectivity of the above-mentioned well-known networks is equal to $3 n-5$ if $n \geqslant 8$.

The remaining of this paper is organized as follows. In Section 2, we give some definitions and lemmas. In Section 3, we study that 2-extraconnectivity of $G\left(G_{0}, G_{1} ; M\right)$. Then, in Section 4, as applications of these results, we determine 2-extraconnectivity of the hypercube $Q_{n}$, the twisted cube $T Q_{n}$, the Möbius cube $M Q_{n}$, the locally twisted cube $L T Q_{n}$ and the crossed cube $C Q_{n}$. Conclusions are in Section 5.

\section{Definitions and lemmas}

For the terminology and notation not defined here, we follow [19]. For a graph $G=(V, E)$ and $S \subset V$ or $S \subset G$, we use $N_{G}(S)$ to denote the set of neighbors of $S$ in $G-S$. For any vertex $v$, we use $d_{G}(x)$ to denote the degree of $x$. A graph $G$ is $k$-regular if $d_{G}(x)=k$ for every $x \in V(G)$. A subset $S \subset V(G)$ is called a vertex-cut if $G-S$ is disconnected. The connectivity of $G$, denoted by $\kappa(G)$, is the minimum cardinality of all vertex-cuts in $G$.

Definition 2.1. Let $G$ be a simple graph. A vertex-cut $S$ of $G$ is called a restricted $h$-vertex-cut if each component of $G-S$ has more than $h$ vertices. The $h$-extraconnectivity of $G$, denoted by $\kappa_{h}(G)$, is defined as $\min \{|S|: S$ is a restricted $h$-vertex-cut of $G\}$ if $G$ has restricted $h$-vertex cuts.

Clearly, when $h=0$, a restricted 0 -vertex-cut is just a vertex-cut. Thus, the concept of the $h$-extraconnectivity is a generalization of the concept of the traditional connectivity. In this paper we explore a special class of graphs $G\left(G_{0}, G_{1} ; M\right)$ proposed by Chen et al. [3].

Definition 2.2. Let $G_{1}$ and $G_{2}$ be two graphs with the same order, and $M$ be any arbitrary perfect matching between vertices of $G_{1}$ and $G_{2}$. The graph $G\left(G_{1}, G_{2} ; M\right)$ is defined as a graph with the vertex-set $V=V\left(G_{1}\right) \cup$ $V\left(G_{2}\right)$ and the edge-set $E=E\left(G_{1}\right) \cup E\left(G_{2}\right) \cup M$.

Lemma 2.3. (See [3].) If both $G_{1}$ and $G_{2}$ are $(k-1)$ regular $(k-1)$-connected graphs with $n$ vertices, then $G\left(G_{1}, G_{2} ; M\right)$ is k-regular k-connected.

Definition 2.4. For a graph $G$, the $c n$-number of $G$, $\operatorname{cn}(G)$, is the smallest integer $\ell$ for which any two vertices in $G$ share at most $\ell$ common neighboring vertices.

From the definition, we have the following result immediately.

Lemma 2.5. $\operatorname{cn}\left(G\left(G_{0}, G_{1} ; M\right)\right)=2$ if $\operatorname{cn}\left(G_{0}\right)=$ $\operatorname{cn}\left(G_{1}\right)=2$.

\section{Main result}

In this section, we present our main result, which can be stated as follows.

Theorem 3.1. Let $G=G\left(G_{0}, G_{1} ; M\right)$. If both $G_{0}$ and $G_{1}$ are $(k-1)$-regular and triangle-free, $\kappa_{1}\left(G_{0}\right)=$ 
$\kappa_{1}\left(G_{1}\right)=2 k-4$, and $c n\left(G_{0}\right)=c n\left(G_{1}\right)=2$, then $\kappa_{2}(G)=3 k-5$ for $k \geqslant 8$ and $\kappa_{2}(G) \geqslant 3 k-5$ for $6 \leqslant k \leqslant 7$.

Proof. By Lemma 2.5, $c n(G)=2$ and so there exist two vertices $x$ and $y$ in $G$ such that $\left|N_{G}(x) \cap N_{G}(y)\right|=$ 2. Let $T=(x, z, y)$ be a path of length 2 between $x$ and $y$. By Lemma 2.3, $G$ is $k$-regular. Since both $G_{0}$ and $G_{1}$ contain no triangles, it is easy to check that $G$ contains no triangles, and so $N_{G}(z) \cap\left(N_{G}(x) \cup N_{G}(y)\right)=\emptyset$. Thus

$\left|N_{G}(T)\right|=(k-1)+(k-2)+(k-2)=3 k-5$.

Let $H=G-\left(T \cup N_{G}(T)\right)$. Since $\kappa_{1}\left(G_{0}\right)=\kappa_{1}\left(G_{1}\right)=$ $2 k-4,|V(G)|=2\left|V\left(G_{1}\right)\right| \geqslant 2\left(\kappa_{1}\left(G_{0}\right)+4\right)=4 k$, and so $V(H) \neq \emptyset$. Since $c n(G)=2,\left|N_{G}(u) \cap N_{G}(T)\right| \leqslant 6$ for any $u \in V(H)$ and $\left|N_{G}(e) \cap N_{G}(T)\right| \leqslant 12$ for any $e \in E(H)$. Since $6<k=\left|N_{G}(u)\right|$ and $12<2 k-2=$ $\left|N_{G}(e)\right|$ for $k \geqslant 8$, there are neither isolated vertices nor isolated edges in $G-N_{G}(T)$. Thus, $N_{G}(T)$ is a restricted 2-vertex-cut of $G$, and so

$\kappa_{2}(G) \leqslant\left|N_{G}(T)\right|=3 k-5$ for $k \geqslant 8$.

We now prove

$\kappa_{2}(G) \geqslant 3 k-5$ for $k \geqslant 5$.

Suppose that there is a subset $F \subset V(G)$ with $|F| \leqslant$ $3 k-6$ such that there are neither isolated vertices nor isolated edges in $G-F$. We want to prove that $G-$ $F$ is connected. To the end, let $F_{0}=F \cap V\left(G_{0}\right)$ and $F_{1}=F \cap V\left(G_{1}\right)$. Clearly, $F_{0} \cap F_{1}=\phi$. Without loss of generality, we may suppose that $\left|F_{0}\right| \geqslant\left|F_{1}\right|$. Then

$\left|F_{1}\right| \leqslant \frac{3 k-6}{2}<\frac{4 k-10}{2}=2 k-5$ for $k \geqslant 5$.

We now prove that $G-F$ is connected by two steps. In Step 1 , we prove that any vertex in $G_{0}-F_{0}$ can be connected to a vertex in $G_{1}-F_{1}$. In Step 2, we prove that $G_{1}-F_{1}$ is connected in $G-F$.

Step 1. Any vertex in $G_{0}-F_{0}$ can be connected to some vertex in $G_{1}-F_{1}$.

Let $u_{0}$ be any vertex in $G_{0}-F_{0}$ and $u_{0} u_{1} \in M$. If $u_{1} \notin F_{1}$, then we are done. So suppose that $u_{1} \in F_{1}$. Since $u_{0}$ is not an isolated vertex in $G-F$, there exist a vertex $v_{0} \in N_{G_{0}}\left(u_{0}\right)-F_{0}$, suppose $v_{0} v_{1} \in M$, if $v_{1} \notin F_{1}$, then we are done, so suppose that $v_{1} \in F_{1}$. Since $u_{0} v_{0}$ is not an isolated edge in $G-F$, there exist a vertex $w_{0} \in N_{G_{0}}\left(u_{0}, v_{0}\right)-F_{0}$. Suppose that $w_{0} w_{1} \in M$, if $w_{1} \notin F_{1}$, then we are done, so suppose $w_{1} \in F_{1}$. The vertex $w_{0}$ may be adjacent to $u_{0}$ or $v_{0}$, without loss of generality, we suppose that $w_{0}$ is adjacent to $v_{0}$, then $N_{G_{0}}\left(w_{0}\right) \cap N_{G_{0}}\left(v_{0}\right)=\emptyset$ and $N_{G_{0}}\left(u_{0}\right) \cap N_{G_{0}}\left(v_{0}\right)=\emptyset$ since $G_{0}$ contains no triangles. And since $\operatorname{cn}\left(G_{0}\right)=2$, so $N_{G_{0}}\left(w_{0}\right) \cap N_{G_{0}}\left(u_{0}\right) \leqslant 2$, thus

$$
\begin{aligned}
\left|N_{G_{0}}\left(u_{0}, v_{0}, w_{0}\right)\right| & \geqslant(k-2)+(k-3)+(k-3) \\
& =3 k-8 .
\end{aligned}
$$

Let $N=N_{G_{0}}\left(u_{0}, v_{0}, w_{0}\right) \cup\left\{u_{0}, v_{0}, w_{0}\right\}$. Then $|N| \geqslant$ $3 k-5$.

Let $M^{\prime}=\{e \in M \mid e$ is incident with a vertex in $N\}$. Then $\left|M^{\prime}\right|=|N| \geqslant 3 k-5$ since $M$ is a perfect matching in $G$. Since $|F| \leqslant 3 k-6$, there exists at least one edge of $M^{\prime}$ whose two end-vertices both are not in $F$. Let $e \in M^{\prime}$ be such an edge. Since $u_{0}, v_{0}, w_{0}$ all are not in $F, u_{0}$ can be connected to $G_{1}-F_{1}$ via the edge $e$.

Step 2. $G_{1}-F_{1}$ is connected in $G-F$.

If there are no isolated vertices in $G_{1}-F_{1}$, then it is clear that $G_{1}-F_{1}$ is connected since $\left|F_{1}\right|<2 k-5<$ $2 k-4=\kappa_{1}\left(G_{1}\right)$ by our hypothesis.

Suppose now that there exists an isolated vertex, say $u_{1}$, in $G_{1}-F_{1}$. Since any two vertices in $G_{1}$ can share at most two common neighbors by our hypothesis, at least $2 k-4$ vertices are to be removed to get two isolated vertices in $G_{1}$. Since $\left|F_{1}\right|<2 k-5$, there is just one isolated vertex $u_{1}$ in $G_{1}-F_{1}$. Let $F_{1}^{\prime}=F_{1} \cup\left\{u_{1}\right\}$. Then $G_{1}-F_{1}^{\prime}$ contains no isolated vertices, and so $G_{1}-F_{1}^{\prime}$ is connected since $\left|F_{1}^{\prime}\right|<2 k-5+1=2 k-4=\kappa_{1}\left(G_{1}\right)$ by our hypothesis. We only need to show that $u_{1}$ can be connected to some vertex in $G_{1}-F_{1}^{\prime}$ via some vertices in $G_{0}-F_{0}$.

Since there are no isolated edges in $G-F, N_{G_{0}}\left(u_{0}\right)-$ $F_{0} \neq \emptyset$. If there is some $v_{0} \in N_{G_{0}}\left(u_{0}\right)-F_{0}$ with $v_{0} v_{1} \in$ $M$ such that $v_{1} \notin F_{1}$, then we are done. So assume $v_{1} \in F_{1}$ for any $v_{0} \in N_{G_{0}}\left(u_{0}\right)-F_{0}$ with $v_{0} v_{1} \in M$. Note $\left|N_{G_{0}}\left(u_{0}, v_{0}\right)\right|=2(k-2)=2 k-4$ since $G_{0}$ contains no triangles. Since $\left|F_{1}\right| \geqslant\left|N_{G_{1}}\left(u_{1}\right)\right|=k-1$ and $\left|F_{0}\right|=|F|-\left|F_{1}\right| \leqslant 3 k-6-(k-1)=2 k-5$, we have $\left|N_{G_{0}}\left(u_{0}, v_{0}\right)\right|>\left|F_{0}\right|$, that is, $N_{G_{0}}\left(u_{0}, v_{0}\right)-F_{0} \neq \varnothing$. If there is some $w_{0} \in N_{G_{0}}\left(u_{0}, v_{0}\right)-F_{0}$ with $w_{0} w_{1} \in$ $M$ such that $w_{1} \notin F_{1}$, then we are done. So assume $w_{1} \in F_{1}$ for any $w_{0} \in N_{G_{0}}\left(u_{0}, v_{0}\right)-F_{0}$ with $w_{0} w_{1} \in$ $M$. Since $G_{0}$ contains no triangles and $\operatorname{cn}\left(G_{0}\right)=2$, $\left|N_{G_{0}}\left(u_{0}, v_{0}, w_{0}\right)\right| \geqslant 3 k-8>2 k-5 \geqslant\left|F_{0}\right|$ for $k \geqslant 4$, that is, $N_{G_{0}}\left(u_{0}, v_{0}, w_{0}\right)-F_{0} \neq \emptyset$. If there is some $x_{0} \in N_{G_{0}}\left(u_{0}, v_{0}, w_{0}\right)-F_{0}$ with $x_{0} x_{1} \in M$ such that $x_{1} \notin F_{1}$, then we are done. So assume $x_{1} \in F_{1}$ for any $x_{0} \in N_{G_{0}}\left(u_{0}, v_{0}, w_{0}\right)-F_{0}$ with $x_{0} x_{1} \in M$. Since $G_{0}$ contains no triangles and $c n\left(G_{0}\right)=2$, it is easy to compute that $\left|N_{G_{0}}\left(u_{0}, v_{0}, w_{0}, x_{0}\right)\right| \geqslant 4(k-1)-6-4=$ $4 k-14$

Let $N=N_{G_{0}}\left(u_{0}, v_{0}, w_{0}, x_{0}\right) \cup\left\{v_{0}, w_{0}, x_{0}\right\}$. Then $|N| \geqslant 4 k-11$. Let $M^{\prime}=\{e \in M \mid e$ is incident with a vertex in $N\}$. Then $\left|M^{\prime}\right|=|N|$ since $M$ is a perfect 
matching in $G$. Since $\left|M^{\prime}\right| \geqslant 4 k-11>3 k-6 \geqslant|F|$ for $k \geqslant 6$, there exists at least one edge of $M^{\prime}$ whose two end-vertices both are not in $F$. Let $e \in M^{\prime}$ be such an edge. Since $u_{0}, v_{0}, w_{0}, x_{0}$ all are not in $F, u_{0}$ can be connected to $G_{1}-F_{1}$ via the edge $e$.

Combining Step 1 with Step 2 fields the inequality (2). It follows from (1) and (2) that $\kappa_{2}(G)=3 k-5$ for $k \geqslant 8$ and $\kappa_{2}(G) \geqslant 3 k-5$ for $6 \leqslant k \leqslant 7$. This completes the proof of the theorem.

\section{Applications}

Topologies of many interconnection networks can be easily viewed as $G\left(G_{0}, G_{1} ; M\right)$ for some $k$-regular graphs $G_{0}$ and $G_{1}$, see Chen et al. [3], such as the hypercube $Q_{n}[11,17]$, the twisted cube $T Q_{n}[1,2]$, the cross cube $C Q_{n}$ [5], the Möbius cube $M Q_{n}$ [4] and the locally twisted cube $L T Q_{n}$ [25]. For these networks, Chen et al. [3] proved that they are super connected and super edge-connected for $n \geqslant 3$; Xu and Wang [23] determined their 1-extraconnectivities and the 1-edgeextraconnectivities both are $2 n-2$ for $n \geqslant 3$; Zhu et al. [29] determined their 2-edge-extraconnectivities all are $3 n-4$ for $n \geqslant 4$. Applying Theorem 3.1, we immediately obtain that their 2-extraconnectivities all are $3 n-5$ for $n \geqslant 8$. The proofs are omitted here for details.

\section{Conclusions}

In this paper, we concentrate on a stronger measure of reliability and fault-tolerance of a network called the 2-extraconnectivity, which not only compensate for shortcoming but also generalize the classical connectivity. For a special class of graphs $G\left(G_{0}, G_{1} ; M\right)$, we explore its 2-extraconnectivity. As applications of our result, we immediately determine the 2-extraconnectivities of some well-known networks, such as the hypercube $Q_{n}$, the twisted cube $T Q_{n}$, the cross cube $C Q_{n}$, the Möbius cube $M Q_{n}$ and the locally twisted cube $L T Q_{n}$, all are $3 n-5$ for $n \geqslant 8$. In other words, for $n \geqslant 8$, at least $3 n-5$ vertices must be removed to disconnect any one of these networks provided that the removal of these vertices does not isolate a vertex or an edge. When these networks are used to model the topological structure of a large-scale parallel processing system, our results provide more accurate measurements for reliability and fault tolerance of the system.

\section{References}

[1] S. Abraham, K. Padmanabhan, An analysis of the twisted cube topology, Proc. International Conference on Parallel Processing 1 (1989) 116-120.
[2] S. Abraham, K. Padmanabhan, The twisted cube topology for multiprocessors: A study in network asymmetry, Journal of Parallel and Distributed Computing 13 (1991) 104-110.

[3] Y.-C. Chen, J.J.M. Tan, L.-H. Hsu, S.-S. Kao, Super-connectivity and super edge-connectivity for some interconnection networks, Applied Mathematics and Computation 140 (2003) 245-254.

[4] P. Cull, S.M. Larson, The Möbius cubes, IEEE Transactions on Computers 44 (5) (1995) 647-659.

[5] K. Efe, A variation on the hypercube with lower diameter, IEEE Transactions on Computers 40 (11) (1991) 1312-1316.

[6] A.H. Esfahanian, Generalized measures of fault tolerance with application to $n$-cube networks, IEEE Transactions on Computers 38 (11) (1989) 1586-1591.

[7] A.-H. Esfahanian, S.L. Hakimi, On computer a conditional edge connectivity of graph, Information Processing Letters 27 (1988) 195-199.

[8] A.-H. Esfahanian, L.M. Ni, B.E. Sagan, The twisted $n$-cube with application to multiprocessing, IEEE Transactions on Computers 40 (1) (1991) 88-93.

[9] J. Fàbrega, M.A. Fiol, Extraconnectivity of graphs with large girth, Discrete Mathematics 127 (1994) 163-170.

[10] F. Haray, Conditional connectivity, Networks 13 (1983) 346357.

[11] F. Harary, J. Hayes, H.J. Wu, A survey of the theory of hypercube graphs, Computers and Mathematics with Applications 15 (4) (1988) 277-289.

[12] A. Hellwig, D. Rautenbach, L. Volkmann, Note on the connectivity of line graphs, Information Processing Letters 91 (1) (2004) $7-10$.

[13] A. Hellwig, L. Volkmann, Sufficient conditions for $\lambda^{\prime}$-optimality in graphs of diameter 2, Discrete Mathematics 283 (2004) 113120.

[14] A. Hellwig, L. Volkmann, Sufficient conditions for graphs to be $\lambda^{\prime}$-optimal, super-edge-connected and maximally edgeconnected, Journal of Graph Theory 48 (2005) 228-246.

[15] J.-X. Meng, Optimally super-edge-connected transitive graphs, Discrete Mathematics 260 (2003) 239-248.

[16] J.-X. Meng, Y.-H. Ji, On a kind of restricted connectivity of graphs, Discrete Applied Mathematics 117 (2002) 183-193.

[17] Y. Saad, M.H. Schultz, Topological properties of hypercubes, IEEE Transactions on Computers 37 (7) (1988) 867-872.

[18] J.-M. Xu, Topological Structure and Analysis of Interconnection Networks, Kluwer Academic Publishers, Dordrecht, Boston, London, 2001.

[19] J.-M. Xu, Theory and Application of Graphs, Kluwer Academic Publishers, Dordrecht, Boston, London, 2003.

[20] J.-M. Xu, M. Lü, On restricted arc-connectivity of regular digraphs, Taiwan Journal on Mathematics 9 (4) (2005) 661-670.

[21] J.-M. Xu, M. Lü, Super connectivity of line graphs and digraphs, Acta Mathematical Applicatal Sinica 22 (1) (2006) 43-48.

[22] J.-M. Xu, M. Lü, Min, M.-J. Ma, A. Hellwig, Super connectivity of line graphs, Information Processing Letters 94 (4) (2005) 191195.

[23] J.-M. Xu, W.-W. Wang, On super or restricted connectivity of some interconnection networks, Ars Combinatoria (2006).

[24] J.-M. Xu, K.-L. Xu, On restricted edge-connectivity of graphs, Discrete Mathematics 243 (1-3) (2002) 291-298.

[25] X.F. Yang, D.J. Evans, G.M. Megson, The locally twisted cubes, International Journal of Computer Mathematics 82 (4) (2005) 401-413.

[26] Z. Zhang, J.-X. Jixiang, Restricted edge-connectivity of edgetransitive graphs, Ars Combinatoria 78 (1) (2006) 297-308. 
[27] Z. Zhang, J.-X. Meng, On optimally- $\lambda^{(3)}$ transitive graphs, Discrete Applied Mathematics 154 (6) (2006) 1011-1018.

[28] Z. Zhang, J.-J. Yuan, A proof of an inequality concerning $k$-restricted edge connectivity, Discrete Mathematics 304 (1-3) (2005) 128-134.
[29] Q. Zhu, J.-M. Xu, M. Lü, Edge fault tolerance analysis of a class of networks, Applied Mathematics and Computation 172 (1) (2006) 111-121. 